\title{
SIMULATION OF AFTERBODY TRANSONIC FLOWS USING PARTIALLY-AVERAGED NAVIER-STOKES MODEL FOR TURBULENCE
}

\section{Deprés and P. Brenner}

EADS ASTRIUM

Route de Verneuil 66 - BP 3200, Les Mureaux 78133, France

\begin{abstract}
This investigation is dedicated to the elaboration of a numerical strategy to simulate accurately and at a reasonable cost the main unsteady features of high Reynolds separated flows. Within this framework, the Partially-Averaged Navier-Stokes (PANS) turbulence model has been used. In this hybrid approach, the modeled-to-resolved scale ratio (the level of physical resolution) is introduced and set explicitly in the closure model of the unresolved scales which is based on the ReynoldsAveraged Navier-Stokes (RANS) paradigm. The flow separation behind an axisymmetric afterbody has been simulated. The large-scale coherent structures are captured and the comparison of the results with wallpressure measurements shows that the flow physics simulated is relevant. Nevertheless, these encouraging results need to be confirmed by deeper investigations on the model behavior and its sensitivity to physical as well as numerical parameters. This step of validation is mandatory before considering an extensive use of the approach at an industrial level.
\end{abstract}

\section{INTRODUCTION}

The presence of a massively separated flow at the base part of a launcher is responsible for strong low-frequency wall-pressure fluctuations, especially during the transonic phase of flight. This aerodynamic excitation can induce a response of the launcher and its subsystems structural modes. With the constant need of improving performance and reliability of launch vehicles, mastering unsteady flow effects and, more specifically, reducing their intensity are of primary importance. The continuous improvement of computing capacities now allows a more extensive use of the numerical tool in the early development of new launchers. This should help providing very early a first estimation of buffet loads and then identifying the potentially 'pathological' configurations to be avoided.

This is an Open Access article distributed under the terms of the Creative Commons Attribution-Noncommercial License 3.0, which permits unrestricted use, distribution, and reproduction in any noncommercial medium, provided the original work is properly cited. 
The choice of a suitable model is a key point to succeed in the simulation of separated flows at high Reynolds numbers. Indeed, classical RANS two-equation models generally fail to predict flow unsteadiness as a one-point closure is not able to represent the large-scale flow physics that depends mainly on the geometry. This is due to an overestimation of the eddy viscosity which damps the natural flow unsteadiness. Detached Eddy Simulation (DES) which uses the best capabilities of both RANS and LES (Large Eddy Simulation) has been shown to be a relevant approach for the computation of separated flows in a great range of applications [1, 2]. However, at an industrial level, its use is still out of reach to treat complex geometrical configurations in an acceptable time limit. In the last decades, various alternatives have been proposed, all referring to some kinds of modified unsteady RANS where the parent RANS model is tuned to take into account the effect of large-scale coherent structures in nonequilibrium flows. This modeling approach was initiated by $\mathrm{Ha}$ Minh who introduced the semideterministic (SDM) approach [3] which was later extended by Hoarau et al. to Organized Eddy Simulation (OES) [4]. More recently, the PANS approach [5, $6]$ has been proposed and identified as promising. Results comparable to DES have been obtained $[5,7]$.

The main objective of this investigation is to evaluate the capability of the PANS approach to predict the unsteady features of separated flows with enough accuracy and at a reasonable cost. The general philosophy of the approach is presented and the main advantages are underlined. The PANS model has been implemented in the FLUSEPA code using a two-equation $k-\varepsilon$ model as a basis and tested on a well documented configuration dealing with an axisymmetric transonic flow separation. Finally, a working logic is discussed to improve the model capabilities and allow a better control of its behavior, especially regarding the demarcation between the resolved and unresolved scales.

\section{TURBULENCE MODELING ADAPTED TO UNSTEADY FLOWS}

The PANS approach is a family of models that are parameterized by modeledto-resolved scale ratios. It is based on the introduction of an arbitrary implicit filter demarcating the resolved and unresolved motion. This is equivalent to define a partial averaging (denoted as $\langle\cdot\rangle$ hereinafter) over a given portion of the fluctuating scales. When a constant filter in space and time is applied to the Navier-Stokes equations, it can be shown that the resulting system of the filtered flow variables is formally identical to the RANS one except the signification of the second-order stress tensor that now refers to the subfilter scale stress. As it does not include the large-scale structures that mostly depend on the geometry, the stress tensor representing the background turbulence is modeled using the RANS 
paradigm. The filtering operation is quantified by two parameters, namely, the unresolved-to-total ratios of kinetic energy and dissipation:

$$
f_{k}=\frac{k_{u}}{k} ; \quad f_{\varepsilon}=\frac{\varepsilon_{u}}{\varepsilon} .
$$

Here $k_{u}$ and $\varepsilon_{u}$ are the unresolved kinetic energy and dissipation, respectively, while $k$ and $\varepsilon$ refer to the RANS quantities resulting from an averaging over all scales of motion. The $f_{k}$ parameter determines the cutoff between the resolved and unresolved scales in the energy spectrum of turbulence. The $f_{\varepsilon}$ parameter indicates the related amount of dissipation that is modeled and, hence, to which extent the dissipation scales overlap the energetic ones. This parameter is a way to control the flow Reynolds number. Note that the turbulence physics imposes $0 \leq f_{k} \leq f_{\varepsilon} \leq 1$ as most of the dissipation occurs at small scales in the energy cascade mechanism. When $f_{k}$ is set to 1 , this corresponds to RANS, whereas for $f_{k}=0$, it is DNS (Direct Numerical Simulation). The amount of flow details that is resolved increases as the $f_{k}$ parameter is decreased provided that the computational grid resolution is adapted to the resolved flow scales.

The model of the unresolved motion is based on a single-point closure and the Boussinesq approximation and, more precisely, on a two-equation model for $k_{u}$ and $\varepsilon_{u}$ quantities (see [6] for the details of derivation):

$$
\begin{aligned}
\langle\rho\rangle \frac{d k_{u}}{d t} & =P_{u}-\varepsilon_{u}+\frac{\partial}{\partial x_{j}}\left(\left(\mu+\frac{\mu_{t u}}{\sigma_{k u}}\right) \frac{\partial k_{u}}{\partial x_{j}}\right) ; \\
\langle\rho\rangle \frac{d \varepsilon_{u}}{d t} & =C_{\varepsilon 1} \frac{P_{u}}{k_{u}}-C_{\varepsilon 2} \frac{\varepsilon_{u}^{2}}{k_{u}}+\frac{\partial}{\partial x_{j}}\left(\left(\mu+\frac{\mu_{t u}}{\sigma_{k u}}\right) \frac{\partial \varepsilon_{u}}{\partial x_{j}}\right) ; \\
\mu_{t u} & =\langle\rho\rangle C_{\mu} \frac{k_{u}^{2}}{\varepsilon_{u}} .
\end{aligned}
$$

The model coefficients are:

$$
C_{\varepsilon 2}=C_{\varepsilon 1}+\frac{f_{k}}{f_{\varepsilon}}\left(C_{\varepsilon 2}-C_{\varepsilon 1}\right) ; \quad \sigma_{k u, \varepsilon u}=\sigma_{k, \varepsilon} \frac{f_{k}^{2}}{f_{\varepsilon}} .
$$

The constants $C_{\varepsilon 1}=1.44 ; C_{\varepsilon 2}=1.92 ; \sigma_{k}=1.0 ; \sigma_{\varepsilon}=1.3$, and $C_{\mu}=0.09$ are those of the classical RANS $k-\varepsilon$ model. The parameterization of the model as a function of two resolution or filter-width control parameters is very convenient as it allows the treatment of a great range of configurations, $f_{k}$ and $f_{\varepsilon}$ being set by the user depending on the flow geometry and the desired accuracy. The smaller the parameters, the more scales are resolved and the more accurate is the simulation. In PANS, the accuracy of LES or DES can be reached although the methods are distinct. However, the main advantage of PANS with regard to 
LES or DES is that the subfilter scale motion is represented by a two-equation model that has been used extensively in the RANS framework and proven to give acceptable results in lots of engineering applications. Hence, in PANS, it is possible to resolve a limited amount of the total kinetic energy depending on the configuration and the desired accuracy whereas in LES, the closure is generally algebraic and the use of a very fine grid resolution extending up to the inertial scales is mandatory.

\section{METHODOLOGY}

\subsection{FLUSEPA Code}

The computations have been carried out using the FLUSEPA code which was first developed for strongly unsteady applications involving bodies in relative motion and propulsive flows such as missile stage separations and takeoffs. Afterward, the field of application quickly extended to any kind of flows. This subsection gives a quick overview of the numerical method. For more details, see $[8,9]$.

The solver is based on a cell-centered finite-volume formulation on unstructured hybrid grids. It integrates the conservation laws for a compressible, turbulent reactive flow loaded with particles and water droplets. The second-order HEUN predictor-corrector explicit scheme is used for time integration. To save computer time, an effective temporal adaptive algorithm is used where each grid cell advances near its maximum allowable time step without degrading accuracy.

The convective fluxes on the cell faces are computed according to the MUSCL (Monotone Upstream Centered Scheme for Conservation Laws) approach of Van Leer. The values at the cell faces are rebuilt from the ones at the center of gravity by supposing linear variations. A correction is then applied to ensure the monotonic behavior of the solution and avoid numerical oscillations near discontinuities. Finally, the Riemann problem is solved through the Godunov algorithm.

The relative motion of the grids is taken into account through an Arbitrarily Lagrangian/Eulerian formulation coupled with a truly conservative Chimera method based on geometrical intersection:

- each body is tied to its own mesh and these meshes overlap each other. The fully covered cells are temporarily excluded from the computation;

- the exact geometrical intersection between the overlapped grid and the overlapping one is computed; and

- the information transfer between meshes is performed by the computation of the fluxes on the intersection area. Since there is no underlying structure 
on the grids, the fluxes between two cells of different meshes (on the intersection area) are processed in the same way as the other fluxes. The only difference is that the volume of the cut cells is variable as the interface with the mask is moving. This technique enforces the balance equations. Thus, the computational method is fully conservative leading to an accurate and robust scheme.

Turbulent flows are modeled with the two equation $k-\varepsilon$ Launder-Sharma model with realizable constraints. The near-wall region can be processed either by a low Reynolds formulation involving damping functions or by a high Reynolds formulation based on standard wall-functions.

\subsection{Test Case}

The test case is extracted from a test campaign that was carried out at $\mathrm{ON}$ ERA in a transonic continuous wind tunnel [10]. The aim of this investigation was the buffet loads induced by flow unsteadiness at transonic speeds behind axisymmetric afterbodies. The configuration selected is shown in Fig. 1. The fully turbulent boundary layer developing on the cylindrical forebody separates at the base corner and is observed experimentally to reattach just before the end of the afterbody. Before separation, the

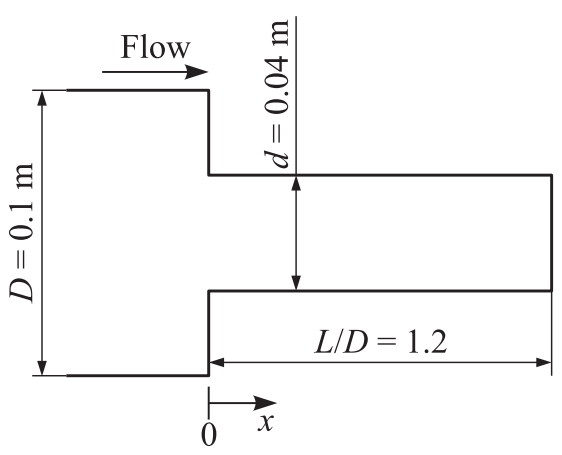

Figure 1 Schematic of the computed test case boundary layer thickness is approximately $0.2 D$.

Steady and unsteady wall-pressure measurements were performed on the cylindrical forebody (before the base corner), the base area, and the rear-body region, both in the streamwise and azimuthal directions. The pressure fluctuations were recorded simultaneously at a sampling rate of $10,240 \mathrm{~Hz}$ during $1.6 \mathrm{~s}$. This allowed a good resolution of the fluctuations regarding the low frequencies of the flow unsteadiness.

\subsection{Computational Grid}

A global view of the computational domain with the boundary condition locations is shown in Fig. 2. The in-flow condition has been set in order to have a boundary layer thickness at separation corresponding to the one measured in 


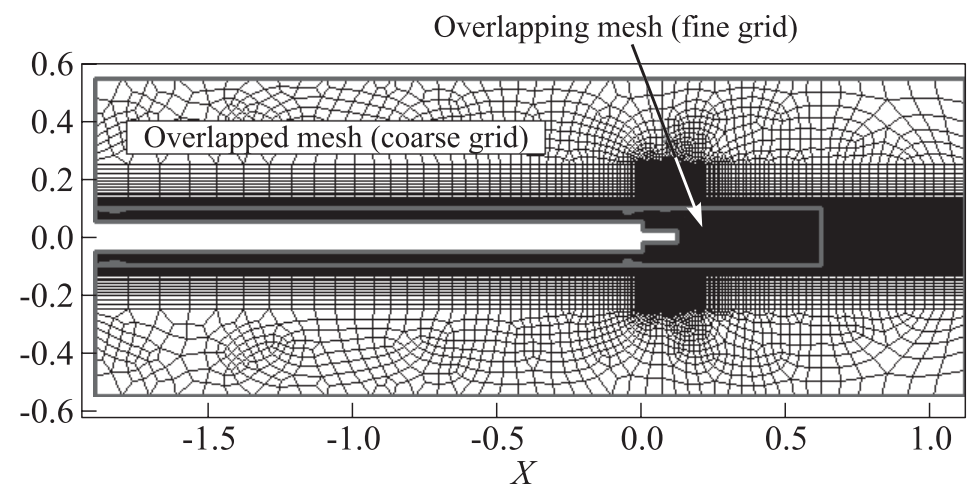

(a)

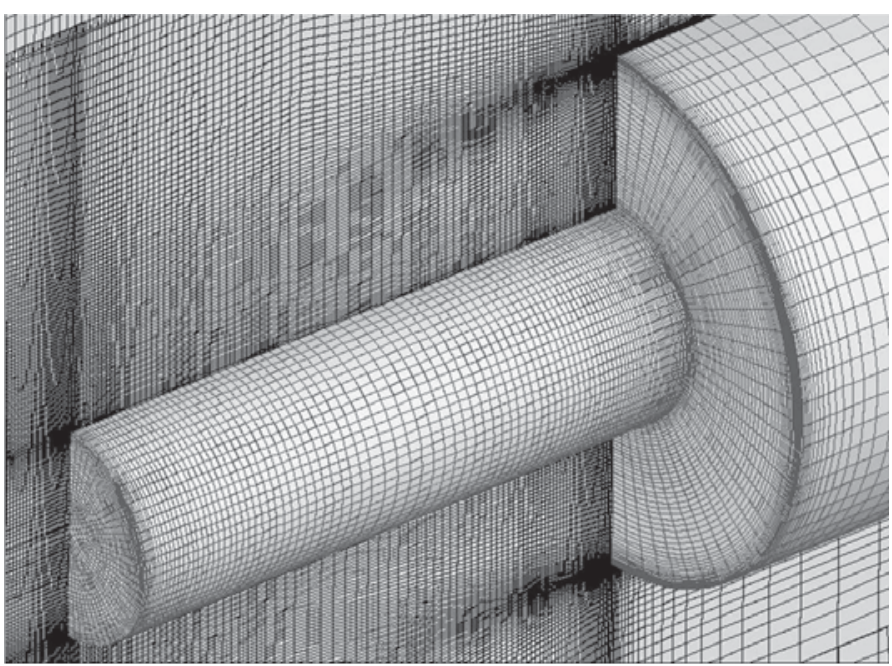

(b)

Figure 2 Global $(a)$ and detailed $(b)$ view of the computational domain

the experiment. A strategy using the overlapping technique of the FLUSEPA code has been applied in order to optimize the mesh refinement in the area of interest and to save computer time. An overlapping fine grid is built around the afterbody including the recirculation region and the near-wake up to $6.5 \mathrm{D}$ downstream of the base. The azimuthal resolution is $5^{\circ}$. This grid is overlapping a coarser one that extends up to the external boundary conditions. The azimuthal resolution is $10^{\circ}$ in this region. A view of the fine mesh in the afterbody region can be seen in Fig. 2. The final computational grid contains nearly $2 \cdot 10^{6}$ hexahedral elements. 


\subsection{Computational Setup}

The computations have been performed with flow conditions derived from the experiments. The Mach number is 0.7 and the Reynolds number based on the diameter is $1.2 \cdot 10^{6}$.

The near-wall region has been treated using wall-functions in order to save computer time by avoiding solving the viscous sublayer. The first near-wall grid point was set to have $y_{+} \approx 22$ just before the boundary layer separation. It is believed here that not integrating the equations up to the wall is not damageable for the simulation accuracy as we are looking for the large-scale unsteadiness of the flow and that, anyway, the fluctuating scales near the wall will never be resolved in such computations.

In PANS, the prescription of the resolution parameters $f_{k}$ and $f_{\varepsilon}$ which define the demarcation between the modeled scales and the resolved one is a crucial issue. It is clear that the computational grid must be fine enough to resolve the targeted amount of flow scales. One must pay attention to this point prior to start the computation since the resolution parameters are independent of the local grid resolution which is not the case in LES or DES.

In this study, a preliminary simulation has been carried out in order to get the first evaluation of the method capability. For the sake of simplicity, spatially constant $f_{k}$ and $f_{\varepsilon}$ fields have been used:

- since the Reynolds number is very high and the flow fully turbulent, $f_{\varepsilon}$ is set to 1 . This is not verified in the near-wall regions where the Reynolds number is low, that is, the dissipation can extend out of the unresolved flow. This point would have to be addressed more thoroughly in a further investigation. It is to be noticed that the use of wall-functions limits the influence of such approximation as the very low-Reynolds region is not computed; and

- the $f k$ value adapted to the present configuration (that is, for a given geometry and grid resolution) has been evaluated by comparing the turbulent scales $\Lambda=k^{1.5} / \varepsilon$ extracted from a preliminary RANS computation and the local grid resolution $\Delta[5]$. The smaller $f_{k}$ value a grid can support can be estimated by

$$
\left(f_{k}\right)_{\min }=\lambda\left(\frac{\Delta}{\Lambda}\right)^{2 / 3} .
$$

This requirement is empirical, the multiplicative constant $\lambda$ being of order 1 but unknown exactly. To avoid underestimating the $f k$ parameter, it has been slightly increased and set finally to 0.4 .

The PANS calculation is initialized using the steady state computed with the RANS $k-\varepsilon$ model. Once the transitory phase is terminated, the simulation is 
performed on a physical duration of $0.16 \mathrm{~s}$ in order to get enough samples for the statistical analysis of the pressure signals. Using the adaptive temporal scheme, a maximum time step of $2.6 \mu$ s could be reached in the biggest cells.

\section{COMPUTATIONAL RESULTS}

The objective of this section is to evaluate to which extent the unsteady flow features are predicted by the PANS method. The deep analysis of the key flow phenomena has already been carried out based on experimental [10] as well as numerical [2] data. This is a challenging configuration due to the complexity of the flow physics. The phenomena are mainly random in nature and the frequency range of fluctuations extends roughly between 0.1 and 0.8 in terms of Strouhal number St $=f D / U_{\infty}$. One can distinguish mainly two features in the spectra:

(1) a somewhat narrow peak at $\mathrm{St} \approx 0.2$. It can be related to the large-scale vortices shed in the axisymmetric wake of the body; and

(2) a broadband peak centered on $\mathrm{St} \approx 0.5-0.6$. This frequency range is related to the smaller and more random coherent structures of the separated shear layer impinging the downstream wall.

\subsection{Static Pressure}

The streamwise distribution of the pressure coefficient $C_{p}=\left(p-p_{\infty}\right) / q_{\infty}$ is given in Fig. 3. The results are plotted for both RANS and PANS computations. The prediction is in good agreement with the experimental data. The recompression

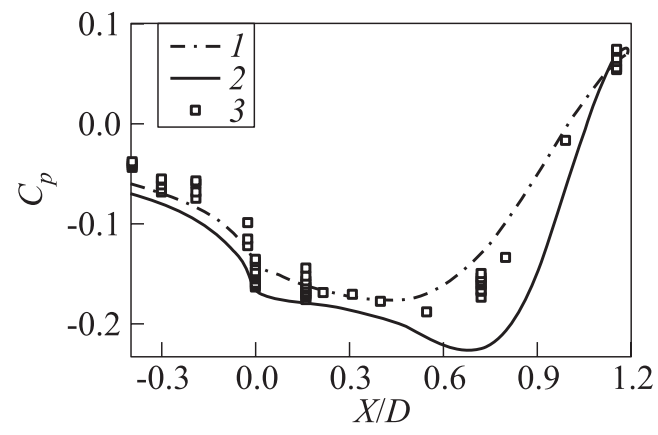

Figure 3 Longitudinal distribution of the static pressure coefficient: 1 - RANS simulation; 2 - PANS simulation; and 3 - experiments 
phase that occurs on the second part of the afterbody is well reproduced. This behavior is related to the mean flow reattachment that occurs just before the end of the afterbody. Nevertheless, one can notice that PANS does not predict the static pressure as accurately as RANS does. This degradation of the results compared to the RANS parent model first suggests that the mean contribution of the large-scale resolved motion is not well reproduced in the unsteady calculation. This point is not well understood for the moment and needs further investigations as the prediction of the mean flow quantities remains an important requirement. Such behavior may be related to the use of spatially constant resolution parameters which are not well suited to compute attached flows, i. e., the boundary layer that develops prior to separation at the base corner.

\subsection{Pressure Fluctuations Levels}

The overall or rms (root-mean-square) wall-pressure fluctuations indicate locally the intensity of the flow unsteadiness. The streamwise distribution of $C_{\text {prms }}=p_{\text {rms }} / q_{\infty}$ along the afterbody is shown in Fig. 4 . The global trend given by the computation is qualitatively good. One can see a steady growth of the fluctuations levels from the separation point. A maximum is reached very next to the end of the afterbody. This maximum is located just upstream of the mean reattachment point location. This is coherent both with the present experimental measurements and the data collected from a numerous configurations of separated flows [11]. Nevertheless, noticeable discrepancies appear in the second part of the recirculation region where the levels are overestimated by a factor 2 at the end of the afterbody. This is probably due to an overrepresentation of the large-scale structures influence. The vortices must be too coherent and their three-dimensionality nature underevaluated. The DES simulations $[2,12]$ are

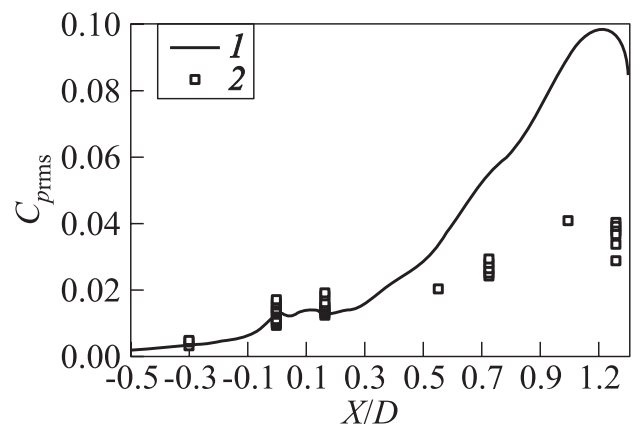

Figure 4 Longitudinal distribution of the unsteady pressure coefficient: 1 - PANS simulation; and 2 - experiments 
found to give a better estimation of the fluctuation levels on the same configuration. In these computations, the grids were noticeably finer and it was shown that azimuthal resolution had a dramatic effect on the results. An accuracy of about $15 \%$ was obtained with $2.5^{\circ}$ between each plane [2] and around $5 \%$ [12] with $1.5^{\circ}$ ( $5^{\circ}$ was retained for the present study). The greater azimuthal resolution probably enhances the mixing thus leading to a greater disorganization and dissipation of the coherent vortices.

\subsection{Autospectral Analysis}

A deeper comparison of the numerical results with the experimental data leads to analyze the frequency range involved in the pressure fluctuations. Power spectral densities are given in Fig. 5. In order to eliminate the discrepancies previously evidenced on the overall fluctuation levels, the spectra are normalized by $p_{\text {rms }}^{2}$. The computed spectra are noisier than the experimental ones because the record

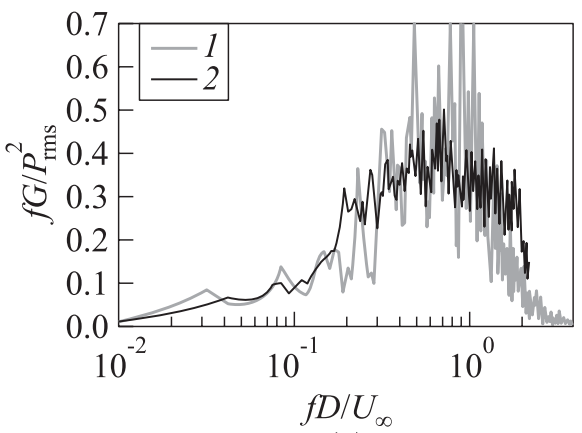

(a)

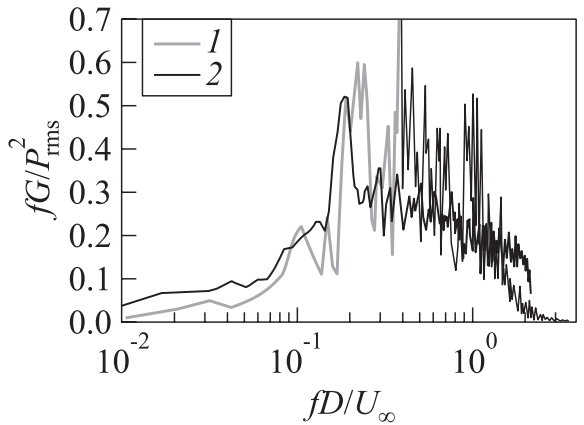

(c)

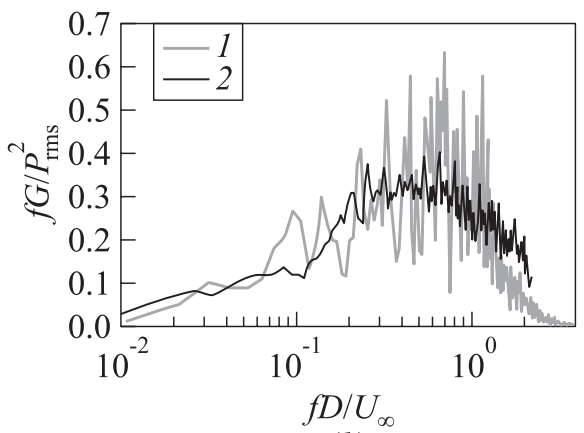

(b)

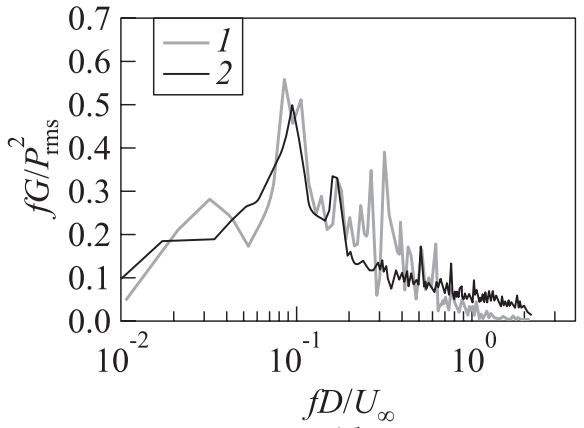

(d)

Figure 5 Pressure spectra $(1$ - PANS simulation; and 2 - experiments $)$ at different longitudinal positions: $(a) X / D=1.155$; (b) 0.99 ; (c) 0,72 ; and $(d) X / D=0$ 
length of the signals is short and the statistics are performed with a limited number of means. It can be seen that the simulation predicts rather well the most energetic frequencies. Moreover, the global shape of the spectra is in good agreement, that is, the main features of the flow unsteadiness are reproduced. The peak at $\mathrm{St} \approx 0.2$ is observed before reattachment at $X / D=0.72$, the exact frequency being somewhat overestimated. On the second part of the rear-body, there is a reinforcement of the broadband fluctuations around $\mathrm{St} \approx 0.6$ due to the interaction of the shear layer with the wall. Next to separation, low-frequency oscillations around $\mathrm{St} \approx 0.1$ are well reproduced. They are attributed to a very slow and large-scale flapping motion of the whole recirculation region.

One can notice that for high frequencies, the decay energy in the signals is steeper in the simulation than in the experiments. This can be interpreted as a cutoff effect which occurs at $\mathrm{St} \approx 1.4$. In principle, this should correspond to the limit between the resolved and unresolved scales set by the $f_{k}$ parameter. As the physical resolution is independent of the grid spacing, a filtering effect due to the mesh is possible although not very probable. Strictly speaking, this hypothesis should only be dismissed by a grid independence study.

\subsection{Coherence Function}

The coherence function between two wall-pressure signals has also been rebuilt as it plays a great part in the unsteady loads exerted by the flow on the body. The module of the coherence function is plotted in Figs. $6 a$ and $6 b$ for sensors separated by a short angle in the azimuthal direction. The correct behavior is obtained although a global overestimation of the coherence levels: the correlations decrease with the increase of both the spatial distance and frequency (spatial filtering of the small-scale fluctuations). The pressure fluctuations are also well predicted at larger distances as shown in Fig. $6 c$ where the phase is also plotted. This demonstrates that the computed propagating phenomena in the recirculation region are representative of the experimental situation.

Finally, such comparison allows to conclude that the computed wall-pressure field is realistic. This ensures that the flow physics simulated is correct even if the contribution of the large-scale structures is somewhat overestimated.

\subsection{Unsteady Flow Details}

No experimental data are presently available in the flowfield. However, one should be interested in assessing the unsteady flow details that are simulated in the present case with the selected resolution. An instantenous numerical Schlieren can be seen in Fig. $7 a$ in the plane of the incoming flow. This view evidences the shear layer that develops from the separation point. One can 


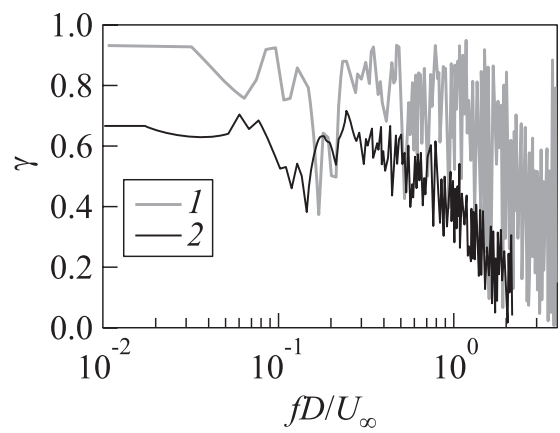

(a)

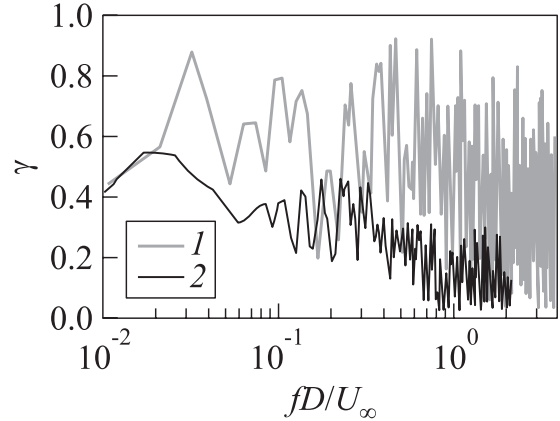

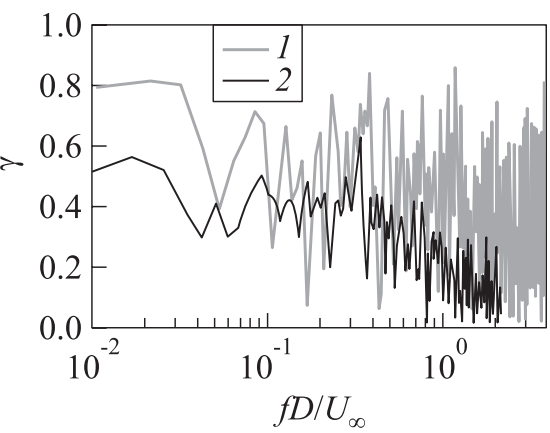

(b)

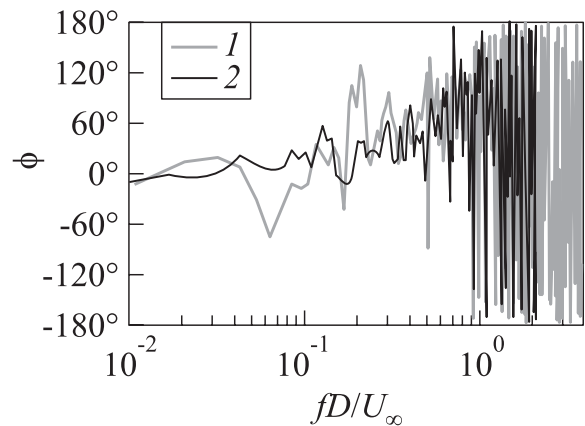

(c)

Figure 6 Coherence function of wall-pressure fluctuations; sensors in the azimuthal direction $(X / D=1.115)(a)$ and $(b)$, and in the longitudinal direction $\left(X_{1} / D\right.$ $\left.=0.99 ; X_{2} / D=1.155\right)(c) ;(a)$ and $(c): 1$ - PANS simulation, $\Delta \theta=10^{\circ}$; and 2 - experiments, $\Delta \theta=11.25^{\circ}$; and $(b): 1-$ PANS simulation, $\Delta \theta=20^{\circ}$; and $2-$ experiments, $\Delta \theta=22.5^{\circ}$

distinguish the presence of small-scale structures that are growing continuously in size as they are advected downstream before impinging the wall. These vortices are responsible for the strong pressure oscillations that occur in the reattachment zone, next to the end of the afterbody.

This analysis does not reveal the three-dimensional (3D) aspects and, therefore, is not fully representative. The use of the $Q$ criterion [13] appears more suitable to reveal the global organization of the flow and, more specially, the coherent vortices as shown in Fig. $7 b$. The iso- $Q$ surface is colored by the axial vorticity. This instantaneous view reveals the complexity of the flow mechanisms as well as the large range of length scales involved in the instabilities. Moreover, it brings out the strong 3D aspect of the flow structure. The initial annular small vortices of the shear layer are progressively disorganized as they approach the reattachment region. At the end of the afterbody, these structures are seen to 


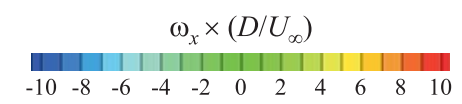

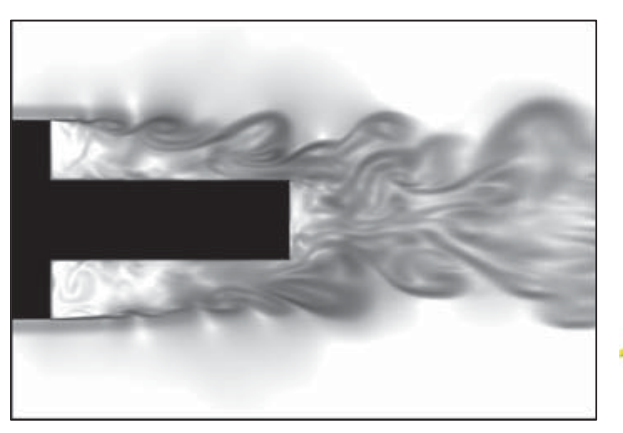

(a)

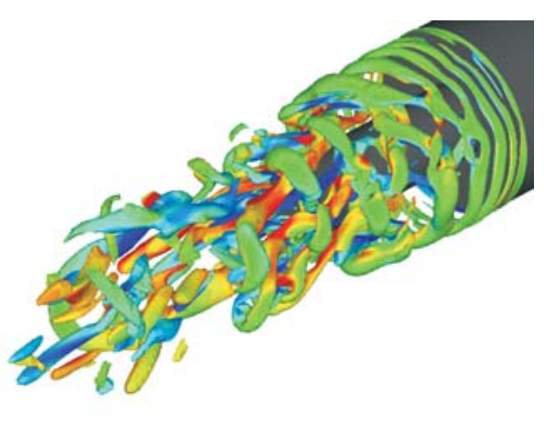

(b)

Figure 7 Flow visualization: numerical Schlieren $(a)$ and isosurface of the $Q$ criterion (b). (Refer Deprés and Brenner, p. 37.)

orientate following the $X$-axis were significant axial vorticity is observed. Several coherent structures can be seen in the near-wake. They are rotating equally in both senses following the $X$-axis as revealed by the high levels of vorticity. This shows that PANS is really able to simulate accurately the large-scale structures of the flow. The complexity of the flow physics suggests that a small value of the $f_{k}$ parameter is required to predict accurately the present configuration.

\section{ON A WORKING LOGIC TO IMPROVE PARTIALLY-AVERAGED NAVIER-STOKES CALCULATIONS}

\subsection{Spatially-Varying Filter-Control Parameters}

The promising results obtained in the simulation of a transonic afterbody flow confirm the potentiality of the PANS approach and then justifies developing further the method. As already mentioned, the preliminary computation was performed by supposing a spatially constant $f_{k}$ distribution over the computational domain. Considering a given configuration, the turbulent scales can change notably with position, for instance, between separation and reattachment. Then, it is clear that a constant $f_{k}$ function is not optimal. Moreover, in such situation, the fixed filter width cannot be strictly valid everywhere in the flow:

- in boundary layers, $f_{k}$ should be equal to 1 as simulating the small scales of an attached flow is still out of reach at high Reynolds numbers. This is 
equivalent to use the parent RANS model in the near-wall regions as DES does. This is fully justified as RANS models are well adapted to predict attached flows; and

- considering the shear layer that forms at the flow separation point, the initial stages cannot be well resolved with an affordable computational grid as the flow mechanism involves small-scale vortical structures resulting from Kelvin-Helmholtz instabilities. Hence, the optimal $f_{k}$ value would also be next to one in these areas.

Therefore, the next step is the extension of PANS to the general case of a spatially-varying $f_{k}$ function adapted to the local turbulent scales and grid resolution, both in the separated and attached regions. Clearly, this will allow resolving more scales in the regions of interest and with a better accuracy. Slowly varying functions compared to that of flow variables will be considered at first. Indeed, it is reminded that in the derivation of the present formalism, a constant implied filter is assumed to commute with spatial and temporal differentiation.

\subsection{Determination of the Optimal Scale Resolution}

From a practical point of view, it is required to have a robust procedure to determine the optimal filter-control parameters $f_{k}$ and $f_{\varepsilon}$. This stage is crucial as a poor estimation of the resolution parameters that can be supported by a given grid should lead to inaccurate solutions. Regarding $f_{\varepsilon}$, it will be generally set to 1 as many industrial applications deal with high Reynolds number flows. Concerning $f_{k}$, a systematic grid independency study would be theoretically the best solution but it is time-consuming and, therefore, not suitable to treat complex configurations. It is more efficient to estimate the local physical resolution using Eq. (1). The turbulent length scale can be extracted from a preliminary RANS calculation and set constant in time all along the PANS simulation [5]. To be robust, this methodology first requires the calibration of the $\lambda$ constant in Eq. (1). It mainly depends on the numerical method but equally on the flows physics simulated. This calibration will be achieved by performing sensitivity studies on suitable test cases to be defined.

\subsection{High-Order Schemes}

To simulate accurately unsteady flows involving coherent structures, the use of high-order numerical schemes is required to avoid filtering the resolved motion and thus modifying the implied cutoff of the simulations. However, most of numerical codes dedicated to the treatment of industrial applications are classically based on two-order accurate numerical schemes. In such conditions, vortical substructures can be poorly resolved due to numerical diffusion as well as dispersive 
effects (sequel of the truncation terms). Thus, the extension of the convective fluxes to the third order of accuracy is under way. An original compact method (the successive corrections method [14]) is used to compute the second derivatives and to correct the first derivatives of conservative variables to a sufficient order of accuracy. This method is based on an iterative process to compute the $(n+1)$ th derivative using the $n$th derivative on the direct neighborhood. This is very suitable for computations on unstructured grids and parallel processing. A piecewise parabolic reconstruction related to a correct numerical quadrature of fluxes on the interfaces lead to the third order of accuracy for the convection terms. A significant improvement of the diffusive fluxes can also be expected on unstructured grids. This will cure the numerical dispersive effects [14] and allow reducing grid-dependency effects on the results.

\section{CONCLUDING REMARKS}

The PANS methodology has been applied successfully to predict the unsteady flow behind an axisymmetric afterbody at transonic speed. The flow physics has been proven to be globally in good agreement with the experimental data. The capability of the approach to compute large-scale flow unsteadiness at a reasonable cost was demonstrated although the pressure fluctuations were noticeably overestimated. This hybrid method, hence, appears as a potential alternative to simulate complex flow configurations dealing with separation and/or wakes that cannot be represented by classical RANS one-point closure models.

In the present investigation, the filter-control parameters were set constant over the computational domain. This approximation cannot be verified in nearwall regions and in the first stages of shear-layers forming at the separation point. This could explain for the discrepancies with the experiment that were evidenced in this study. To confirm and consolidate these preliminary results, it is planned to extend the method to slowly-varying $f_{k}$ functions in order to adapt locally the physical resolution. Furthermore, this could allow treating near-wall regions in RANS (that is, modeling all the scales) as in DES. It is believed that this could enhance significantly the accuracy of the simulations. Moreover, it opens the possibility to compute efficiently a great range of engineering applications in a zonal way by delimiting the regions to be treated in RANS or in PANS.

\section{ACKNOWLEDGMENTS}

CNES (the French Space Agency) is warmly acknowledged for co-funding this numerical study. The experimental database was obtained within the framework 
of the ATAC (Aerodynamics of Nozzles and Afterbodies) program funded by CNES and ONERA.

\section{REFERENCES}

1. Squires, K.D., J.R. Forsythe, S.A. Morton, W.Z. Strang, K. E. Wurtzler, R.F. Tomaro, M. J. Grismer, and P. R. Spalart. 2002. Progress on detached-eddy simulation of massively separated flows. AIAA Paper No. 2002-1021.

2. Deck, S., and P. Thorigny. 2007. Unsteadiness of an axisymmetric separatingreattaching flow: Numerical investigation. Phys. Fluids 19.

3. Ha Minh, H. 1990. Physique et modélisation des écoulements turbulents compressibles. 27th Colloquium AAAF d'Aérodynamique Appliqu'ee. Marseille, France.

4. Hoarau, Y., M. Braza, P. Rodes, G. Tzabiras, C. Allain, E. Berton, D. Favier, and C. Maresca. 2002. Turbulence modelling of unsteady flows with a pronounced periodic character. IUTAM Symposium on Turbulence Modelling of Unsteady Periodic Flows. Toulouse, France.

5. Girimaji, S.S., and K.S. Abdol-Hamid. 2005. Partially averaged Navier-Stokes model for turbulence: Implementation and validation. AIAA Paper No. 2005-502.

6. Girimaji, S. S. 2006. Partially-averaged Navier-Stokes model for turbulence: A Reynolds-averaged Navier-Stokes to direct numerical simulation bridging method. J. Appl. Mech. 73:413-21.

7. Basu, D., A. Hamed, and K. Das. 2005. DES, hybrid RANS/LES and PANS models for unsteady separated turbulent flow simulations. FEDSM'05 Proceedings. Houston, Texas, USA.

8. Pollet, M., and P. Brenner. 1989. Aerodynamics with moving bodies applied to solid propulsion. AIAA Paper No. 89-2779.

9. Brenner, P., and M. Pollet. 2005. Highly unsteady flows about bodies in relative motion. 40éme Colloque AAAF d'aérodynamique appliquée. Toulouse, France.

10. Deprés, D., P. Reijasse, and J.P. Dussauge. 2004. Analysis of unsteadiness in afterbody transonic flows. AIAA J. 42:2541-50.

11. Mabey, D. G. 1972. Analysis and correlation of data on pressure fluctuations in separated flow. J. Aircraft 9(9):642-45.

12. Weiss, P.-E., S. Deck, J.-C. Robinet, and P. Sagaut. 2009. On the dynamics of axisymmetric turbulent separating/reattaching flows. Phys. Fluids 21.

13. Dubief, Y., and F. Delcayre. 2000. On coherent-vortex identification in turbulence. J. Turbulence 1(1):1-22.

14. Haider, F. 2009. Discrétisation en maillage non structuré général et application LES. Ph.D. Thesis. UPMC-Paris. 